\title{
AVALIAÇÃO DO DESEMPENHO LOGÍSTICO DO TRANSPORTE RODOVIÁRIO DE MADEIRA UTILIZANDO REDE DE PETRI EM UMA EMPRESA FLORESTAL DE MINAS GERAIS ${ }^{1}$
}

\author{
Raiane Ribeiro Machado ${ }^{2}$, Márcio Lopes da Silva ${ }^{3}$, Carlos Cardoso Machado $^{3}$ e Helio Garcia Leite ${ }^{3}$
}

\begin{abstract}
RESUMO - Este trabalho teve por objetivo utilizar o modelo de avaliação do desempenho logístico de cadeias produtivas (ADELCAP) centrado nos tempos dos elementos do ciclo operacional do transporte de madeira, visando otimizar os recursos, garantir um fluxo contínuo de abastecimento e assegurar um volume adequado de entrada de madeira na fábrica. O ciclo operacional de transporte de madeira é composto pelo carregamento de madeira no campo, viagem carregada, descarregamento, garagem e viagem vazia. Para tanto, utilizou-se como estudo de caso uma empresa produtora de celulose do Estado de Minas Gerais. Nesse modelo de cadeia logística de transporte de madeira, utilizou-se a Rede de Petri Temporal, por trabalhar com dois tempos que correspondem a uma duração de sensibilização. Criaram-se cenários com as mesmas variações nas duas regionais estudadas e realizaram-se simulações com os softwares JARPe ARP, a fim de avaliar o desempenho operacional e econômico. As variações aplicadas nos cenários foram: melhoria das estradas florestais; aumento do número de carregadores; e melhoria da eficiência da garagem. Em ambas as regionais, o cenário com as três variações (Cenário 7) apresentou a maior redução nos tempos dos ciclos de transporte de madeira em relação à situação atual, sendo 19,24 e 21,48\%, respectivamente, para Rio Doce e Cocais das Estrelas. Considerando-se apenas como custo operacional o valor pago pelo frete, não estando incluso o custo referente a investimentos, tanto na regional do Rio Doce quanto em Cocais das Estrelas, ele apresentou maior redução no cenário 7 (20,09 e $21,22 \%$, respectivamente).
\end{abstract}

Palavras-chave: Logística, redes de Petri e ciclo operacional de transporte de madeira.

\section{EVALUATION OF LOGISTIC PERFORMANCE OF WOOD HAULING USING PETRI NET IN A FOREST INDUSTRY OF MINAS GERAIS}

\begin{abstract}
The present work aimed at using the model of logistic performance evaluation of productive chains (ADELCAP) centered in the operational cycle time elements of wood transport, seeking to optimize the resources, to guarantee a continuous flow of supplying and to assure an appropriate volume of wood entrance in the industry. The wood transport cycle time consists of wood loading, wood hauling, wood unloading, garage and wood unhauling. A cellulose producing company in the State of Minas Gerais was used as case study. Initially, the logistics and supply chain of the company was diagnosed to know the main existent obstacles along the chain; defining their preliminary vary key parameters, based on the characterization of their structure and operation; finally, the main directors that condition their efficiency and competitiveness were identified and evaluated. The model of chain logistics of wood transport used the Temporal Petri Net for working with two times that correspond to a sensitization period. Their properties are as follows: simulation (working with
\end{abstract}

\footnotetext{
${ }^{1}$ Recebido em 02.09.06 e aceito para publicação em 25.11.2006.

${ }^{2}$ Departamento de Administração da Universidade do Estado do Mato Grosso. E-mail: <raianemachado@terra.com.br>.

${ }^{3}$ Departamento de Engenharia Florestal da Universidade Federal de Viçosa, 36570-000 Viçosa-MG. E-mail: <marlosil@ufv.br>.
} 


\begin{abstract}
hypothetical scenarios), dynamic (exit values depend on the entrance values); determinist (exit variables are not random); continuous (time is measured by real numbers); and temporal in the transitions. Scenarios, with the same variations in the two wood-producing regionals were created, and simulations with the JARP and ARP software were carried out to evaluate the operational and economic performance. The variations applied to the scenarios were: improvement of the forest roads; increase of loader numbers, and improvement of garage efficiency. For both regionals, the scenario with the three variations (Scenario 7) presented the largest reduction in cycle times of wood transport in relation to the current situation, being $19.24 \%$ and $21.48 \%$, respectively, for Rio Doce and Cocais das Estrelas. The analyzed operational cost is the value paid for the freight, not including the investment costs. Both regionals, Rio Doce and Cocais das Estrelas, presented a larger reduction in the Scenario 7 , of $20.09 \%$ and $21.22 \%$, respectively.
\end{abstract}

Keywords: Logistic, Petri Nets and log transportation cycle time.

\section{INTRODUÇÃO}

É crescente o destaque do setor florestal brasileiro para a economia nacional. Assiste-se por todo o mundo, em diferentes tipos de organizações, o surgimento de um interesse renovado pelas atividades florestais, das quais se destaca a produção madeireira (NOCE et al., 2005). A economia florestal brasileira tem participação significativa nos indicadores socioeconômicos do país, como o Produto Interno Bruto (PIB), empregos, salários, impostos e balança comercial. No mercado internacional de produtos florestais como a celulose, madeira, móveis, laminados etc., o Brasil vem conquistando espaço em razão das vantagens competitivas que possui (VALVERDE et al., 2003). Um fator de real significado que influi no resultado final do empreendimento florestal é a sua localização com relação às fontes consumidoras, por causa do custo de transporte (COLOMBELLI FILHO, 1973). O transporte é o elemento mais importante do custo logístico para a grande maioria das empresas transportadoras, pois o frete costuma absorver cerca de 60\% do gasto logístico (TABOADA, 2002). A madeira é um produto de valor específico relativamente baixo, ou seja, o volume transportado é muito elevado e o valor da carga é baixo, e isso torna o transporte um dos principais problemas na empresa florestal (SEIXAS, 1992).

O maior entrave para a completa e extensa difusão da logística nas empresas brasileiras é a falta de uma cultura de trabalho com enfoque integral. As empresas têm trabalhado durante décadas utilizando um enfoque vertical, por funções, o que era válido nas condições do ambiente de negócios do passado. As novas exigências, que o ambiente competitivo impõe às organizações, pregam a adoção de um enfoque integral, por objetivos, sistêmico, em que o melhor para cada

R. Árvore, Viçosa-MG, v.30, n.6, p.999-1008, 2006

\footnotetext{
R. Árvore, Viçosa-MG, v.30, n.6, p.999-1008, 2006
}

parte não significa necessariamente o melhor para todo o sistema. Outra dificuldade consiste na carência de informações contábeis que sirvam para apoiar a correta tomada de decisões logísticas. Os sistemas de custeio convencionais oferecem os seus resultados orientados principalmente para os produtos, e a logística precisa de informações contábeis referentes às atividades. A falta de hábito de trabalho com parceiros constitui outro problema. As exigências competitivas levam a estabelecer relacionamentos de parceria, tanto com fornecedores de produtos e serviços logísticos, quanto com os próprios clientes da empresa. Relações essas que permitem um funcionamento mais eficiente dos processos logísticos em que as empresas estão envolvidas. Outra grande dificuldade está na falta de ferramentas para avaliar o desempenho da logística. Há carência de ferramentas, com enfoque estruturado, para realizar uma eficiente e eficaz avaliação do desempenho dos processos logísticos (TABOADA, 2002).

A teoria das Redes de Petri nasceu da tese de doutorado de Carl Adam Petri na Technical University of Darmstadt (Alemanha), em 1962, intitulada "Comunicação com autômatos". Alguns anos depois, um grupo de pesquisadores do MIT lançou as bases do que se tornaram as "Redes de Petri”. A Rede de Petri é uma ferramenta de modelagem matemática e gráfica com atividades concorrentes e assíncronas que permitem a análise de sistemas complexos de produção, os quais são difíceis de serem modelados por técnicas tradicionais, como Filas de Espera, Cadeias de Markov e Redes Pert, entre outras.

Os objetivos do trabalho foram testar as potencialidades da Rede de Petri como ferramenta de modelagem e análise da cadeia logística de suprimento rodoviário de madeira e avaliar o seu desempenho operacional. 


\section{MATERIAL E MÉTODOS}

\subsection{Caracterização das regionais produtoras de madeira estudadas}

Os trabalhos foram desenvolvidos em áreas pertencentes a uma empresa de celulose em Minas Gerais, onde se utilizam veículos de transporte rodoviário de madeira até a unidade consumidora (fábrica). As regiões estudadas foram Cocais das Estrelas (CE) e Rio Doce (RD), pertencentes à regional produtora de Belo Oriente. As regionais dispunham de 4 e 30 composições veiculares de carga (CVC) para o transporte de madeira, além de 1 e 3 carregadores florestais, respectivamente em Rio Doce e Cocais das Estrelas.

O Quadro 1 ilustra o total de área plantada com eucalipto, a produção anual média por regional e a distância média de transporte rodoviário das regionais estudadas até a indústria.

O tempo do ciclo operacional de transporte rodoviário de madeira depende da quantidade de carregadores florestais no campo, do número de veículos de transporte rodoviário, das condições das estradas, da distância de transporte, da velocidade média de operação etc. (Figura 1). Vale salientar que, no pátio da fábrica, foram utilizados dois carregadores frontais, para o descarregamento nas esteiras dos picadores 3 e 4, e três gruas para movimentação de madeira no pátio.

\subsection{Composições Veiculares de Carga}

O sistema de transporte rodoviário florestal utilizado é terceirizado. A CVC utilizada é o rodotrem (Figura 2), destinado ao transporte de toras longitudinais, em quatro feixes de 4,40 m de comprimento, totalizando uma carga útil média de 45 toneladas e um peso bruto total combinado (PBTC) médio de 74 toneladas.

\subsection{Coleta de dados e Amostragem}

Os dados de tempo dos elementos do ciclo operacional, custos e movimentação de madeira no pátio foram coletados da Central de Logística da empresa em duas etapas. Trabalhou-se com dados dos ciclos coletados no período de julho a novembro de 2005 . Foi utilizada a amostragem casual simples, sendo o número de ciclos operacionais estimado através da metodologia utilizada por Barnes (1968). O número de ciclos empregados foram de 67 e 38, respectivamente para Rio Doce e Cocais das Estrelas. Inicialmente, realizouse um estudo-piloto do ciclo operacional, buscando estabelecer o número mínimo de ciclos para cada uma das regionais estudadas, para um erro de amostragem admissível fixado em 5\%, a 95\% de probabilidade. Os tempos de viagem carregada e descarregada foram ponderados em relação à distância média percorrida e aos tempos de carregamento, descarregamento e garagem, em relação ao tempo total do ciclo. O desviopadrão (sigmas) são faixas normalmente adotadas para a definição da variabilidade dos processos nas empresas. A fim de reduzir a margem de erro nos cenários atual e hipotéticos, trabalhou-se com três sigmas, e isso significa que pelo menos $99,73 \%$ dos dados coletados estão dentro do padrão de qualidade.

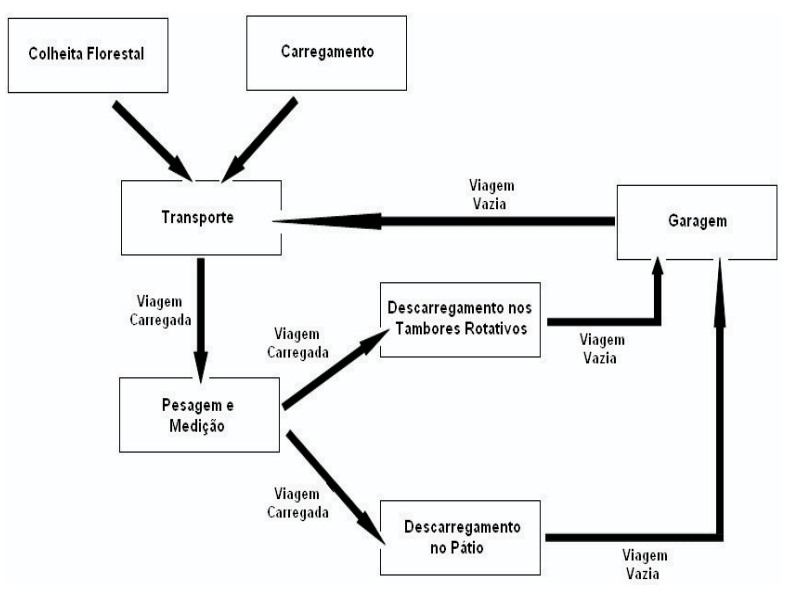

Figura 1 - Descrição da cadeia logística do transporte rodoviário de madeira.

Figure 1-Description of the chain logistics of the road wood transport.

Quadro 1 - Distribuição da produção de madeira, área plantada e distância da indústria. Table 1 -Distribution of the wood production, planted area and distance of the industry

\begin{tabular}{ccccc}
\hline Regional & $\begin{array}{c}\text { Produção Média de } \\
\text { Madeira - 2005 (ton.) }\end{array}$ & $\begin{array}{c}\text { Área Plantada } \\
\text { (ha) }\end{array}$ & $\begin{array}{c}\text { Distância Média } \\
\text { Total (km) }\end{array}$ & $\begin{array}{c}\text { Distância Média } \\
\text { Estradas Florestais (km) }\end{array}$ \\
\hline Rio Doce & 300.000 & 18.756 & 20 & 6,2 \\
Cocais das Estrelas & 210.000 & 11.092 & 83 & 46,4 \\
\hline
\end{tabular}

Fonte: CENIBRA. 


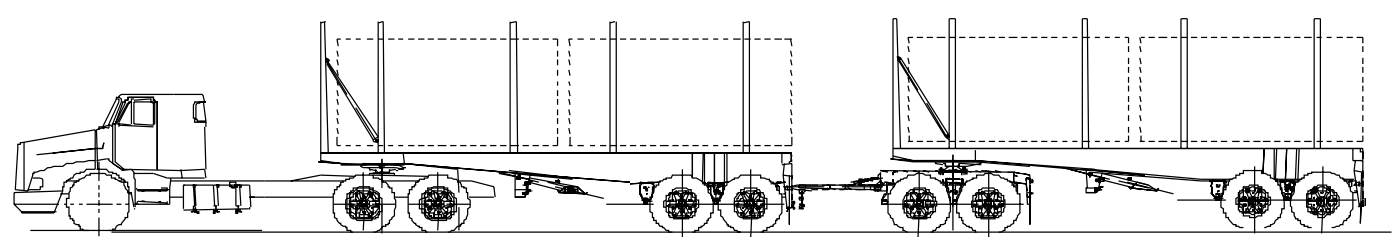

Figura 2 - Rodotrem para o transporte de quatro feixes de toras de 4,40 m.

Fonte: Stein et al. (2001) Figure 2-Rodotrem for the transport of four bunches of logs of $4.40 \mathrm{~m}$.

\subsection{Aplicação do modelo ADELCAPe dos programas ARP e JARP}

O modelo ADELCAP de avaliação do desempenho logístico de cadeias produtivas e os programas ARP e JARP foram utilizados como ferramentas de simulação, análise e avaliação de desempenho da cadeia de suprimento rodoviário de madeira. Para avaliar seu desempenho, foram utilizados os tempos dos elementos do ciclo operacional do transporte de madeira. Na avaliação de desempenho, com o ARP, utilizou-se a abordagem orientada a eventos. Nesse modo foram definidos um evento (transição) inicial e até 10 eventos-destino. As transições foram disparadas, sendo as medidas estatísticas efetuadas entre o disparo do evento inicial e o disparo de algum evento-destino, medindo-se o tempo médio entre eventos e a probabilidade de sua ocorrência. Após essa etapa, procedeu-se a simulação computacional, utilizando a Rede de Petri como ferramenta de apoio à decisão em nível operacional.

Para subsidiar a análise da estrutura e dos componentes da cadeia logística foram obtidas informações na empresa e visitas a campo e pátio da indústria. A estrutura da cadeia logística foi modelada no programa JARP, porém a análise estrutural não foi realizada devido às limitações do programa ARP.

Foi realizado um diagnóstico da cadeia de suprimento rodoviário de madeira, com ênfase ao seu desempenho, quanto aos tempos de ciclo operacional. O estágio atual (características, procedimentos e tecnologia empregados) de desenvolvimento da cadeia, bem como outros recursos de natureza logística, foram conhecidos e avaliados em relação ao desempenho em termos de tempo de ciclo operacional. Assim, tratou-se de estruturála graficamente conforme a Figuras 3, e a legenda para compreensão está descrita no Quadro 2.

Elaboraram-se diferentes cenários de funcionamento da cadeia de suprimento rodoviário de madeira para aplicação do modelo concebido (Quadro 3). A definição do problema requer quantificar o tempo necessário para que uma quantidade de madeira percorra a cadeia logística, desde o carregamento no campo até chegar à fabrica, bem como quantificar os recursos necessários para manter o sistema funcionando sob determinados padrões tecnológicos e de nível de serviço.

Em cada cenário, em ambas as regionais foi avaliado o desempenho logístico, em termos de tempo de ciclo operacional e utilização de frota, associado a cada cenário simulado, em contraposição à situação atual da cadeia logística. Para conhecer o impacto na cadeia logística, oriundo das modificações propostas em cada cenário, foram consideradas as variáveis essenciais (elementos do ciclo operacional de transporte) e a frota de caminhões vinculada a cada regional.

\section{RESULTADOS E DISCUSSÃO}

Uma vezque oADELCAPé um modelodeterminístico, com base no tempo do ciclo operacional, no qual as incertezas não são consideradas, situações atípicas, como madeira transportada para a indústria, mas descartada no pátio por não-conformidade, não são captadas, pois esse tipo de aleatoriedade não é reconhecido pelo modelo. Apesar disso, alguns fatores de ordem prática podem influenciar o tomador de decisão a optar por outra estrutura diferente daquela que possibilitaria a redução dos tempos de ciclo operacional do transporte rodoviário de madeira. O tempo do ciclo de cada cenário detalhado por elemento pode ser conferido nos anexos.

\subsection{Modelagem e simulação dos cenários para a regional do Rio Doce}

\subsubsection{Cenário atual}

O cenário que representa a atual forma de operação do sistema modelado de transporte de madeira, da regional do Rio Doce para a fábrica, está representado por parte da Rede de Petri (Figura 3), através dos lugares (RD $)$ e transições $\left(T_{R D}\right)$, e a partir dele se processaram as simulações dos cenários hipotéticos. A definição da estrutura adotada como cenário atual só foi possível após uma visita à empresa. 
Quadro 2 - Cont Table 2-Cont.

\begin{tabular}{|c|}
\hline Legenda \\
\hline Lugares: \\
\hline $\mathrm{CE}_{6}=$ Veículo na fábrica pronto para ser descarregado \\
\hline $\mathrm{CE}_{7}=$ Madeira sendo descarregada do veículo \\
\hline $\mathrm{CE}_{8}=$ Veículos prontos para se deslocarem para garagem \\
\hline $\mathrm{CE}_{9}=$ Veículo vazio a caminho da garagem \\
\hline $\begin{array}{l}\mathrm{CE}_{10}=\text { Veículo vazio pronto para se deslocar até madeira pronta } \\
\text { na estrada }\end{array}$ \\
\hline $\mathrm{CE}_{11}=$ Carregador livre para iniciar carregamento \\
\hline $\mathrm{MD}_{1}=$ Madeira descarregada na fábrica \\
\hline $\mathrm{DE}_{1}=$ Carregador livre para iniciar descarregamento \\
\hline Elementos do ciclo operacional: \\
\hline $\mathrm{TRD}_{1}=$ Início do carregamento \\
\hline $\mathrm{TRD}_{2}=$ Carregamento do veículo \\
\hline $\mathrm{TRD}_{3}=$ Início da viagem carregada até a fábrica \\
\hline $\mathrm{TRD}_{4}=$ Viagem carregada até a fábrica e pesagem do veículo \\
\hline $\mathrm{TRD}_{5}=$ Início do descarregamento \\
\hline $\mathrm{TRD}_{6}=$ Descarregamento do veículo \\
\hline $\mathrm{TRD}_{7}=$ Início do deslocamento para garagem \\
\hline $\mathrm{TRD}_{8}=$ Veículo na garagem \\
\hline $\mathrm{TRD}_{9}=$ Viagem vazia até madeira pronta \\
\hline $\mathrm{TCE}_{10}=$ Início do carregamento \\
\hline $\mathrm{TCE}_{11}=$ Carregamento do veículo \\
\hline $\mathrm{TCE}_{12}=$ Início da viagem carregada até a fábrica \\
\hline $\mathrm{TCE}_{13}=$ Viagem carregada até a fábrica e pesagem do veículo \\
\hline $\mathrm{TCE}_{14}=$ Início do descarregamento \\
\hline $\mathrm{TCE}_{15}=$ Descarregamento do veículo \\
\hline $\mathrm{TCE}_{16}=$ Início do deslocamento para garagem \\
\hline $\mathrm{TCE}_{17}=$ Veículo na garagem \\
\hline $\mathrm{TCE}_{18}=$ Viagem vazia até madeira pronta \\
\hline $\begin{array}{l}\text { A cada elemento do ciclo operacional existe um intervalo } \\
\text { de disparo associado (tempos). Os símbolos } \theta_{1} \text { e } \theta_{2} \text { representam, } \\
\text { os valores mínimo e máximo de tempo de cada intervalo. }\end{array}$ \\
\hline Lugares e transições (RD) são da Regional do Rio Doce \\
\hline Lugares e transições (CE) são da Regional de Cocais das Estrelas. \\
\hline
\end{tabular}

\subsubsection{Cenários hipotéticos}

Dentre as três variáveis estudadas nos cenários, a que mais interfere no resultado final do ciclo operacional do Rio Doce é o aumento do número de carregadores (cenário 2). Isso porque a distância percorrida em estradas florestais e o tempo de permanência na garagem são pequenos em relação ao tempo de carregamento do veículo. Conseqüentemente, os melhores cenários hipotéticos são aqueles formados pela combinação do aumento de número de carregadores e outra atividade (Quadro 4). O tempo total gasto para cumprir o transporte de toda carga diária é distribuído entre todos os veículos da regional.

R. Árvore, Viçosa-MG, v.30, n.6, p.999-1008, 2006

\subsection{Modelagem e simulação dos cenários para a regional de Cocais das Estrelas}

\subsubsection{Cenário atual}

O cenário que representa a atual forma de operação do sistema modelado de transporte de madeira, da regional de Cocais das Estrelas para a fábrica, está representado por parte da Rede de Petri (Figura 3) através dos lugares $\left(\mathrm{CE}_{\mathrm{x}}\right)$ e transições $\left(\mathrm{TCE}_{\mathrm{x}}\right)$, e a partir dele se processaram as simulações dos cenários hipotéticos. A definição da estrutura adotada como cenário atual só foi possível após uma visita à empresa.

\subsubsection{Cenários hipotéticos}

Dentre as três variáveis estudadas nos cenários, a que mais interfere no resultado final do ciclo operacional de Cocais das Estrelas é a melhoria das estradas florestais (cenário 1), isso porque o tempo de carregamento do veículo e a permanência na garagem são pequenos em relação ao tempo de deslocamento do veículo. Conseqüentemente, os melhores cenários hipotéticos são aqueles formados pela combinação da melhoria das estradas florestais e outra atividade (Quadro 5). O tempo total gasto para cumprir o transporte de toda carga diária é distribuído entre todos os veículos da regional.

\subsection{Avaliação de desempenho dos cenários}

$\mathrm{Na}$ avaliação de desempenho do transporte rodoviário de madeira, com Rede de Petri Temporal (RDP), utilizou-se a função de densidade de probabilidade uniforme associada ao intervalo de disparo de cada transição, com abordagem orientada a estados.

Analisando os resultados de desempenho do transporte de madeira na regional do Rio Doce, obtidos na simulação com o ARP, verificou-se que, dentre os três fatores variáveis dos cenários, o que interfere mais significativamente é o número de carregadores, como pode ser visto no cenário $2(10,59 \%)$, seguido das melhorias das estradas florestais (cenário 1) que apresentou bom resultado $(7,40 \%)$. Mas o impacto causado pela melhoria da eficiência da garagem (cenário 3) é pouco expressivo (3,97\%). Individualmente, o melhor cenário é o número 2 , porém os demais cenários, que são a combinação dos três primeiros, apresentam resultados melhores. Os cenários 4, 6 e 7 exibem os 
melhores resultados, por serem combinações com o cenário 2. Na Regional do Rio Doce, o cenário 7 apresentou o melhor desempenho (19,24\%). Na Figura 4 , apresentam-se os resultados da simulação dos cenários e respectivos desempenhos. Observou-se, nessa figura, a redução causada nos tempos dos ciclos de transporte de madeira em relação ao cenário atual, sendo possível notar que os cenários com melhores resultados são combinações com os cenários 1 e 2, para Rio Doce e Cocais das Estrelas, respectivamente.

\subsection{Avaliação do desempenho operacional do transporte rodoviário de madeira}

Para se avaliar o desempenho operacional do transporte rodoviário de madeira, em função da carga transportada, distância percorrida e tempo de ciclo, foram calculados os desempenhos em cada cenário simulado. Observou-se que, quanto maior o tempo do ciclo operacional, pior o desempenho operacional do transporte de madeira (Figura 5 e Quadro 6).

Quadro 3 - Descrição dos cenários hipotéticos estudados Table 3 - Description of the studied hypothetical scenarios

\begin{tabular}{|c|c|c|}
\hline Cenários & Descrição & Impacto no tempo do ciclo \\
\hline Atual & $\begin{array}{l}\text { Situação real das estradas florestais, do número } \\
\text { de carregadores e da eficiência da garagem }\end{array}$ & \\
\hline$\overline{\text { Cenário } 1}$ & Melhoria das estradas florestais & $\begin{array}{l}\text { Redução em } 50 \% \text { do tempo de viagem em } \\
\text { estradas florestais (ida e volta) }\end{array}$ \\
\hline Cenário 2 & Aumento do número de carregadores & $\begin{array}{l}\text { Redução em } 50 \% \text { do tempo de carregamento } \\
\text { através do aumento de carregadores (Rio Doce } \\
\text { de } 1 \text { para } 2 \text { ou Cocais das Estrelas de } 3 \text { para } 6 \text { ) }\end{array}$ \\
\hline Cenário 3 & Aumento da eficiência na garagem & $\begin{array}{l}\text { Redução em } 50 \% \text { do tempo de permanência } \\
\text { na garagem }\end{array}$ \\
\hline Cenário 4 & $\begin{array}{l}\text { Melhoria das estradas florestais e aumento } \\
\text { do número de carregadores }\end{array}$ & $\begin{array}{l}\{\text { Redução em } 50 \% \text { do tempo de viagem em } \\
\text { estradas florestais (ida e volta) }\}+\{\text { Redução em } \\
50 \% \text { do tempo de carregamento através do } \\
\text { aumento de carregadores (Rio Doce de } 1 \text { para } 2 \\
\text { ou Cocais das Estrelas de } 3 \text { para } 6)\}\end{array}$ \\
\hline Cenário 5 & $\begin{array}{l}\text { Melhoria das estradas florestais e aumento } \\
\text { da eficiência na garagem }\end{array}$ & $\begin{array}{l}\text { Redução em 50\% do tempo de viagem em } \\
\text { estradas florestais (ida e volta) }\}+\{\text { Redução em } \\
50 \% \text { do tempo de permanência na garagem }\} \\
\end{array}$ \\
\hline Cenário 6 & $\begin{array}{l}\text { Aumento no número de carregadores e da } \\
\text { eficiência na garagem }\end{array}$ & $\begin{array}{l}\text { \{Redução em 50\% do tempo de carregamento } \\
\text { através do aumento de carregadores (Rio Doce de } \\
1 \text { para } 2 \text { ou Cocais das Estrelas de } 3 \text { para } 6)\}+ \\
\{\text { Redução em } 50 \% \text { do tempo de permanência } \\
\text { na garagem }\}\end{array}$ \\
\hline Cenário 7 & $\begin{array}{l}\text { Melhoria das estradas florestais, aumento } \\
\text { do número de carregadores e aumento da } \\
\text { eficiência na garagem }\end{array}$ & $\begin{array}{l}\{\text { Redução em } 50 \% \text { do tempo de viagem em } \\
\text { estradas florestais (ida e volta) }\}+\{\text { Redução em } \\
50 \% \text { do tempo de carregamento através do } \\
\text { aumento de carregadores (Rio Doce de } 1 \text { para } 2 \\
\text { ou Cocais das Estrelas de } 3 \text { para } 6)\}+\{\text { Redução } \\
\text { em } 50 \% \text { do tempo de permanência na garagem }\}\end{array}$ \\
\hline
\end{tabular}

Quadro 4 - Resultados dos tempos médios por ciclo e total da Regional do Rio Doce

Table 4-Results of the mean times per cycle and total of Rio Doce Regional

\begin{tabular}{cccc}
\hline Cenários & Tempo Médio por Ciclo (min.) & Tempo Médio Total (min.) & Tempo Médio Total (horas) \\
\hline Atual & 214 & 4708 & $78: 28$ \\
C1 & 199 & 4378 & $72: 58$ \\
C2 & 192 & 4224 & $70: 24$ \\
C3 & 208 & 4576 & $76: 16$ \\
C4 & 177 & 3894 & $64: 54$ \\
C5 & 193 & 4246 & $61: 46$ \\
C6 & 186 & 4092 & $68: 12$ \\
C7 & 171 & 3762 & $62: 42$ \\
\hline
\end{tabular}


Quadro 5 - Resultados dos tempos médios por ciclo e total da Regional de Cocais das Estrelas Table 5 - Results of the mean times per cycle and total of Cocais das Estrelas Regional

\begin{tabular}{cccc}
\hline Cenários & Tempo Médio por Ciclo (min.) & Tempo Médio Total (min.) & Tempo Médio Total (horas) \\
\hline Atual & 469 & 7504 & $125: 04$ \\
C1 & 408 & 6528 & $108: 48$ \\
C2 & 440 & 7040 & $117: 20$ \\
C3 & 460 & 7360 & $122: 40$ \\
C4 & 379 & 6064 & $101: 04$ \\
C5 & 399 & 6384 & $106: 24$ \\
C6 & 431 & 6896 & $114: 56$ \\
C7 & 370 & 5920 & $98: 40$ \\
\hline
\end{tabular}

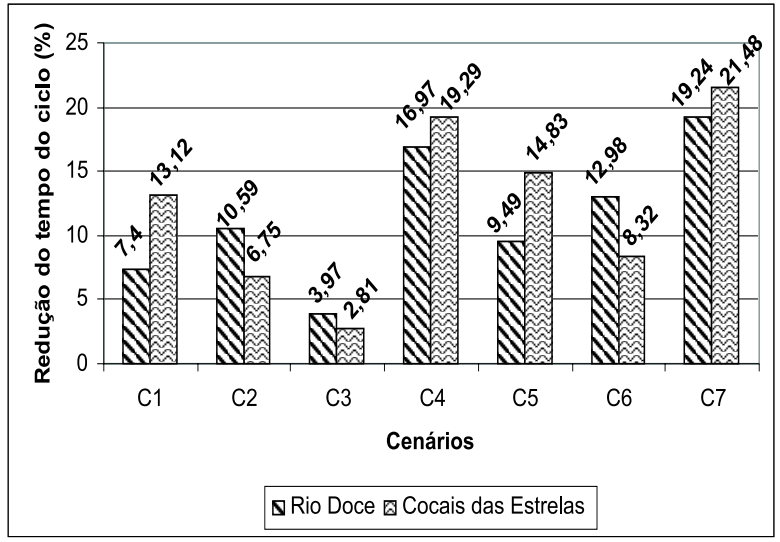

Figura 4 - Redução dos tempos dos ciclos de transporte de madeira em relação ao cenário atual.

Figure 4-Reduction of cycle times of wood transport in relation to the current scenario.

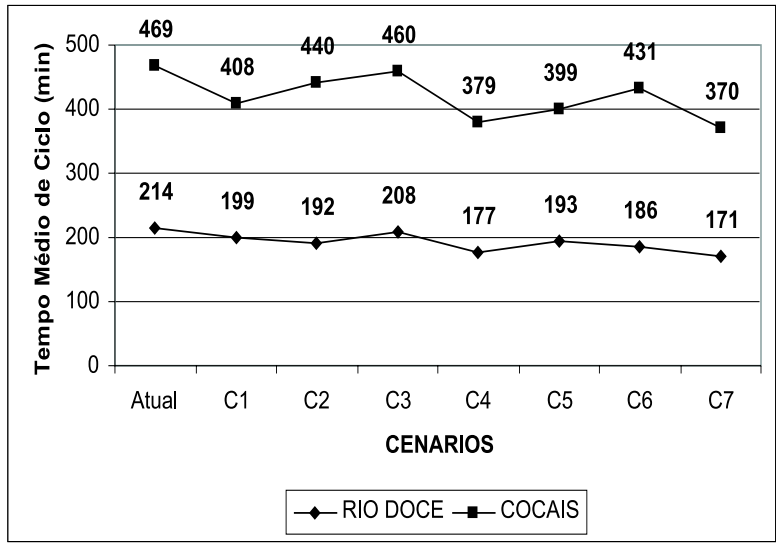

Figura 5 - Comportamento dos tempos dos ciclos operacionais dos cenários simulados.

Figure 5-Behavior of operational cycle times of the simulated scenarios.

Quadro 6 - Resultados simulados de desempenho do transporte de madeira (t/km.h)

Table 6 - Simulated results of operational performance of the wood transport $(t / \mathrm{km} . \mathrm{h})$

\begin{tabular}{cccccccc}
\hline & & Rio Doce & & & \multicolumn{3}{c}{ Cocais das Estrelas } \\
\cline { 2 - 3 } \cline { 7 - 8 } Cenários & Mínimo & Médio & Máximo & & Mínimo & Médio & Máximo \\
\hline Atual & 1,9565 & 0,6308 & 0,3750 & & 0,1740 & 0,0694 & 0,0434 \\
1 & 2,1094 & 0,6784 & 0,4018 & & 0,2099 & 0,0797 & 0,0494 \\
2 & 2,4545 & 0,7031 & 0,4091 & & 0,1768 & 0,0739 & 0,0467 \\
3 & 1,9853 & 0,6490 & 0,3868 & & 0,1749 & 0,0707 & 0,0444 \\
4 & 2,7000 & 0,7627 & 0,4412 & & 0,2140 & 0,0858 & 0,0538 \\
5 & 2,1429 & 0,6995 & 0,4154 & & 0,2112 & 0,0815 & 0,0507 \\
6 & 2,5000 & 0,7258 & 0,4232 & & 0,1778 & 0,0755 & 0,0479 \\
7 & 2,7551 & 0,7895 & 0,4576 & & 0,2154 & 0,0879 & 0,0553 \\
\hline
\end{tabular}

O tempo total de viagem (ida + retorno) representa cerca de 39,25 e 68,66\% do tempo do ciclo de transporte de madeira, respectivamente, nas regionais do Rio Doce e de Cocais das Estrelas. Embora a velocidade média de operação em ambas as regionais sejam praticamente iguais (Cocais das Estrelas $=29 \mathrm{~km} / \mathrm{h}$ e Rio Doce $=$ $27 \mathrm{~km} / \mathrm{h}$ ), o desempenho operacional do transporte de madeira em Cocais das Estrelas foi inferior ao do
Rio Doce, devido ao fato de distância média ser quatro vezes menor, portanto mais sensível à melhoria da qualidade das estradas (Figura 6).

\subsection{Avaliação do desempenho econômico do transporte rodoviário de madeira}

O custo operacional utilizado é o valor pago pelo frete, não estando incluso o custo referente a 
investimentos. Na regional do Rio Doce, pode-se observar que o cenário 7 não só apresentou o melhor desempenho em termos de tempo do ciclo, como também o menor custo operacional por ciclo, ou seja, uma redução de $20,09 \%$ no custo do frete da madeira entregue à fábrica em relação ao custo atual. O impacto nos custos operacionais por ciclo na regional do Rio Doce varia de 2,80\% ( $\$ 223,93)$ no cenário 3 a $20,09 \%$ ( $\mathrm{R} \$ 184,10)$, no cenário 7. Na regional de Cocais das Estrelas, assim como no Rio Doce, pode-se observar que o cenário 7 não só apresentou o melhor desempenho em termos de tempo do ciclo, como também no custo operacional por ciclo. Isso representa uma redução de $21,22 \%$ no custo do frete da madeira

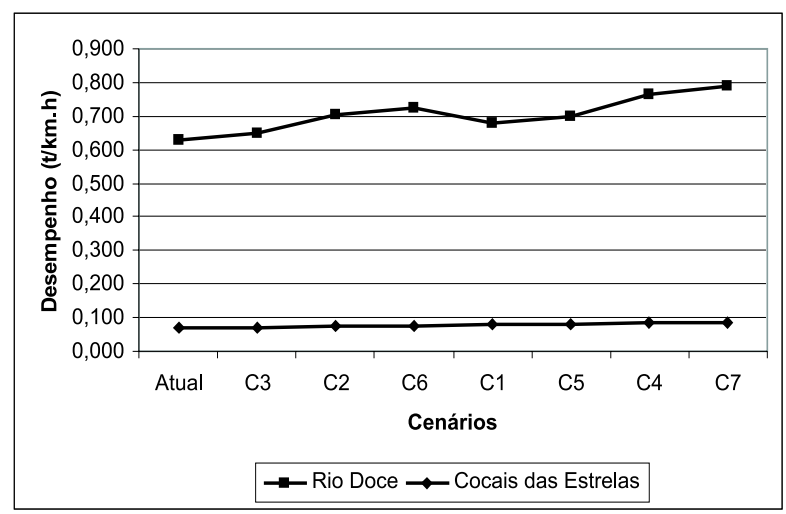

Figura 6-Evolução do desempenho médio simulado do transporte de madeira nos diferentes cenários.

Figure 6-Evolution of the simulated mean performance of wood transport in the different scenarios.

\section{CONCLUSÕES}

- Comprovaram-se as potencialidades da Rede de Petri Temporal como ferramenta de modelagem, simulação, análise e avaliação de desempenho da cadeia logística de suprimento rodoviário de madeira.

- Foi possível diagnosticar o atual nível de desenvolvimento logístico do transporte de madeira estudado através do modelo desenvolvido, utilizandose Rede de Petri Temporal.

- Os fatores de maior influência no desempenho operacional do transporte de madeira nas áreas estudadas foram: o aumento no número de carregadores para Rio Doce e melhoria das estradas florestais para Cocais das Estrelas. entregue na fábrica em relação ao custo atual. O impacto nos custos operacionais por ciclo na regional de Cocais das Estrelas variou de 2,06\% (R $\$ 672,66)$ no cenário 3 até $21,22 \%(\mathrm{R} \$ 541,05)$ no cenário 7 .

Os custos operacionais do veículo, por ciclo de transporte de madeira na regional do Rio Doce, em relação ao cenário atual, é menor nos cenários onde houve aumento do número de carregadores, ou seja, cenários 4, 6 e 7. Os custos operacionais do veículo, por ciclo de transporte de madeira na regional de Cocais das Estrelas em relação ao cenário atual, sendo menores nos cenários onde houve melhorias nas estradas (cenários 4, 5 e 7), apresentaram os melhores resultados (Figura 7).

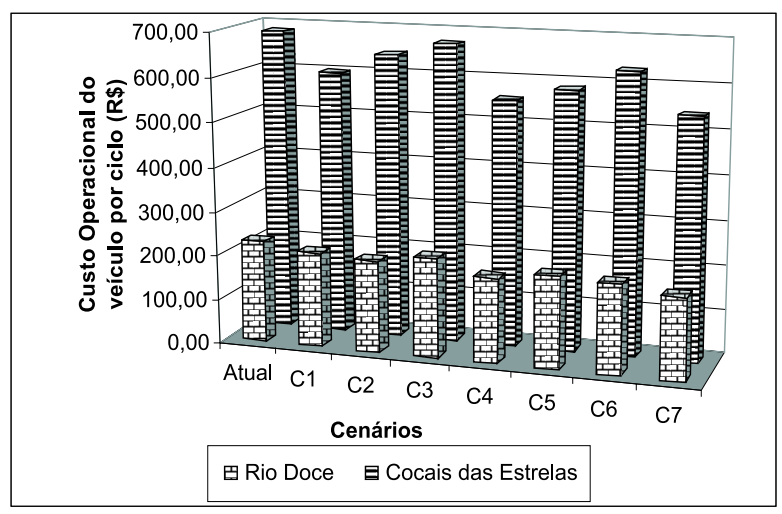

Figura 7 - Custo operacional do veículo por ciclo nos cenários de cada regional.

Figure 7-Operational cost of the vehicle per cycle in the scenarios of each regional.

- As melhorias propostas através dos cenários possibilitaram uma redução no tempo do ciclo operacional de até 19,24 e 21,48\%, respectivamente em Rio Doce e Cocais das Estrelas.

- É possível uma redução de custo operacional de transporte de madeira de até 20,09 e 21,22\%, respectivamente em Rio Doce e Cocais das Estrelas, não se considerando os custos de investimento;

- É viável reduzir em até $12 \%$ o custo da madeira posta na fábrica.

\section{REFERÊNCIAS BIBLIOGRÁFICAS}

BARNES, R.M. Motion and time study: design and measurement of work. 6.ed. New York: John Willey \& Sons, 1968. 799p.

R. Árvore, Viçosa-MG, v.30, n.6, p.999-1008, 2006 
COLOMBELLI FILHO, M. Efeito do custo do transporte no preço da madeira. In: SEMINÁRIO PAULISTA DE SILVICULTURA, 1.; 1973,

Campinas. Anais... Campinas: 1973. p. 98-108.

NOCE, R. et al . Análise de risco e retorno do setor florestal: produtos da madeira. Revista Árvore, v. 29, n. 1, p. 77-84, 2005

SEIXAS, F. Uma metodologia de seleção e dimensionamento da frota de veículos rodoviários para o transporte principal de madeira. 1992. 106 f. Tese (Doutorado em Engenharia de Transportes) Universidade de São Carlos, São Carlos, 1992.
STEIN, F. R.; RODRIGUES, L. A.; SCHETTINO, S. Sistema de transporte rodoviário da Celulose Nipo Brasileira - CENIBRA. In: SIMPÓSIO BRASILEIRO SOBRE COLHEITAE TRANSPORTE FLORESTAL, 5.; 2001, Porto Seguro. Anais... Porto Seguro: Sociedade de Investigações Florestais, 2001. p. 109-121.

TABOADA, C. M. Logística: o diferencial da empresa competitiva. Revista FAE Business, n.2, p. 4-8, 2002.

VALVERDE, S.R. et al . Efeitos multiplicadores da economia florestal brasileira. Revista Árvore, v. 27, n. 3, p. 285-293, 2003. 